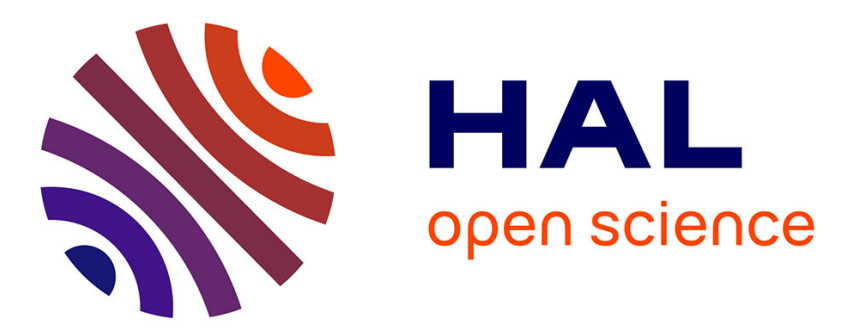

\title{
Algebraic estimation in partial derivatives systems: parameters and differentiation problems
}

\author{
Rosane Ushirobira, Anja Korporal, Wilfrid Perruquetti
}

\section{To cite this version:}

Rosane Ushirobira, Anja Korporal, Wilfrid Perruquetti. Algebraic estimation in partial derivatives systems: parameters and differentiation problems. Alban Quadrat; Eva Zerz. Algebraic and Symbolic Computation Methods in Dynamical Systems, 9, Springer, pp.183-200, 2020, Advances in Delays and Dynamics. hal-01617321

\section{HAL Id: hal-01617321 \\ https://hal.inria.fr/hal-01617321}

Submitted on 16 Oct 2017

HAL is a multi-disciplinary open access archive for the deposit and dissemination of scientific research documents, whether they are published or not. The documents may come from teaching and research institutions in France or abroad, or from public or private research centers.
L'archive ouverte pluridisciplinaire HAL, est destinée au dépôt et à la diffusion de documents scientifiques de niveau recherche, publiés ou non, émanant des établissements d'enseignement et de recherche français ou étrangers, des laboratoires publics ou privés. 


\title{
Algebraic estimation in partial derivatives systems: parameters and differentiation problems
}

\author{
Rosane Ushirobira, Anja Korporal, Wilfrid Perruquetti
}

\begin{abstract}
Two goals are sought in this paper: namely, to provide a succinct overview on algebraic techniques for numerical differentiation and parameter estimation for linear systems and to present novel algebraic methods in the case of several variables. The state-of-art in the introduction is followed by a brief description of the methodology in the subsequent sections. Our new algebraic methods are illustrated by two examples in the multidimensional case. Some algebraic preliminaries are given in the appendix.
\end{abstract}

\section{Introduction}

Many challenging questions in signal processing and control involve the estimation of derivatives of measured time signals, usually in noisy environment. This important issue is known as a numerical differentiation. Several approaches were proposed on this subject, based on different frameworks in applied mathematics and engineering. In control theory, designing a differentiator is an important problem, with many applications [2, 6, 17]. Some classical solutions are based on the leastsquares polynomial interpolation and provide good offline results for this matter, see for example [15]. On another groundwork, just to mention a few works, numerical differentiators defined on an observer design basis were proposed in [4,6,13, 14, 41]

Rosane Ushirobira

Inria, Non-A team, 40 avenue Halley, 59650 Villeneuve d'Ascq, France e-mail: Rosane.

Ushirobiralinria.fr

Anja Korporal

Inria, Non-A team, 40 avenue Halley, 59650 Villeneuve d'Ascq, France e-mail: Anja. Korporaleinria.fr

Wilfrid Perruquetti

École Centrale de Lille \& CRIStAL (UMR CNRS 9189) \& Université Lille Nord de France \& Non-A, Inria, France e-mail: Wilfrid.Perruquettieinria.fr 
and digital filter processing techniques used in [1, 3, 32, 37]. A high-order sliding mode based differentiator is designed in [8] by developing the results from [16] and it provides very satisfactory estimations despite some chattering in the response. We may also remark that the homogeneous finite-time-differentiator defined in [29] presents no chattering, but it is more sensitive to the signal amplitude.

The problem of estimating parameters in linear systems appears in the mathematical modeling of a physical phenomena. Differential equations in the considered model may contain parameters that are simply difficult to determine through data collecting, perhaps due to noisy measurements. This essential problem has attracted the attention of researchers in many fields. For instance, parameter estimation is a central subject in statics inference and several procedures can be applied to this problem, such as the maximum likelihood. Also, parameter estimation problems are also often related to optimization techniques.

There are countless works on numerical differentiation and parameter estimation. Among the recent advances on these issues, a promising solution is provided by differential algebra and operational calculus tools. This algebraic branch was initiated in the works by M. Fliess and H. Sira-Ramírez [10]. A clear description of the procedure, containing many useful explanations, can be found in [9, 19]. In despite of the innovative character of this framework, this algebraic approach remains quite underused. Nevertheless, some works do apply these ideas, see for instance [22, 42, 43] and for more practical developments, see for example [24, 27, 50]. For more details, the reader may refer to a quite extensive survey recently published in [39].

Algebraic methods within the numerical differentiation context were first applied to the univariable numerical differentiation by Mboup et al. in [20] where the authors use Jacobi projections to construct estimators for the derivatives. As described by the authors, the key idea of the method in this latter is to consider the $n$ th-derivative of a smooth signal at a point $\tau$ as a single parameter to be estimated from a noisy observation of the signal. From that, a pointwise derivative can be estimated by varying $\tau$. A truncated Taylor series expansion of the signal is the starting point in this technique, and the computations are then made in the operational domain. A slight drawback in the approximation by a truncated Taylor polynomial model may be its ephemeral character. To reduce this fast transient behavior, an improvement of the technique was proposed in [33]. A through study of these algebraic estimators, with emphasis on the error analysis, can be found in [18]. In that work, estimators based on fractional derivatives were introduced. An interesting computer architecture to accelerate the computation of the aforesaid algebraic derivative estimator was implemented in [28] using reconfigurable logic and implemented in an FPGA (field-programmable gate array).

In the multidimensional case, the estimation of derivatives of a noisy signal concern also many problems in engineering. For instance, in economy issues, in addition to the fields of signal processing or control. To tackle this numerical differential problem, most likely more problematic than the unidimensional case, several techniques were developed. The most commonly used is the finite differences method. The instability of possible solutions to these problems arise from the presence of noise due to the differentiation. 
The use of algebraic tools for multidimensional numerical differentiation was addressed by Riachy et al. in [34-36]. Their inspiration comes from the original ideas in [10] and from the solutions proposed by [20].

The algebraic method developed in this paper is motivated by the parameter estimation methods elaborated in [45-47]. In those works, Weyl algebra based tools grant the estimation of amplitudes, signal and frequencies of a sinusoidal signal, providing faster estimates than known methods [5, 12, 48] (simulated examples in [12] provide fast estimates, however in more than a fraction of the period). The main advantage of our method is to give closed formulas for derivatives and parameter estimates. Furthermore, algebraic estimation techniques strongly rely on differential elimination. So, a number of different estimators (i.e. appropriate differential operators providing estimates) can be devised for a given estimation problem (this is well illustrated through a change-point detection problem in [21]). Hence, it appears that the quality of an estimator varies markedly with the order of the selected differential operators used in the elimination. The Weyl Algebra point of view introduced here within the algebraic context allows to characterize and to select the minimal order operators associated to any given estimation problem. Finally, let us stress that all algebraic approaches mentioned above in this Introduction share a very useful characteristic: obtained estimates are integrals of the noisy measured signal, so these integrals act as time varying filters.

Section 2 starts with a general introduction of the procedure of algebraic estimation, followed by the presentation of two estimation problems: numerical differentiation and parameter identification. In Section 4, the algebraic estimation of derivatives is illustrated through a significant example. To expose our method on a multidimensional parameter estimation problem, a particular partial differential equation was examined. It is the example of the heat conduction on a thin rod that is discussed and treated by algebraic estimations (this type of equation was considered in [38], also based on algebraic techniques). Proposed solutions to this problem in the algebraic framework are given in Section 5. The Appendix contains generalities on algebraic structures, as well as useful properties for the algebraic methods.

\section{Problem formulation}

As mentioned in the Introduction, numerous engineering problems concern the estimation of state variables or parameters. In this section, we describe briefly how algebraic methods proceed, in general, to this estimation.

Most of the time, the mathematical modeling of physical phenomena provide a description of the aforementioned practical problems through a differential equations framework. States or parameters to be identified appear in the terms of these differential equations.

Roughly speaking, for such a given differential equations system, algebraic methods observe typically the following sequence of steps: 
1. Passage to the operational domain through the Laplace transform or by using Mikusinski operational calculus [25 26 49]]: thanks to this step, differential equations are converted into algebraic ones, consequently allowing algebraic concepts to be applied. The resulting algebraic expressions depend on the Laplace variable $s$.

2. Computations with the algebraic equations using structural properties: in this part, the aim is to apply algebraic tools on the equations in order to find expressions and closed formulas for the parameters or derivatives estimations.

3. Return to the time domain and identification: the algebraic expressions in the Laplace variable $s$ found in the previous step are converted into the time domain through the inverse Laplace transform. Possibly a time dependent system on the parameters must be solved.

It is notably in Step 2. that the advantages among different algebraic approaches can be seen. Indeed, in the differential elimination necessary in this process, structural properties of differential algebra are useful. Most of these procedures result in estimates given by integrals (rather than derivatives) of the noisy measured signal and these integrals will then provide noise attenuation. Thanks to special forms for the annihilators (differential operators involved in the differential elimination) developed in the appendix, the identification process presented in this paper will result in faster and less noise sensitive estimates. Therefore, these particular annihilators allow a better choice of suitable differential operators allowing the elimination of problematic parameters, or yield a more convenient matrix representation that will ease the solution of a system.

\subsection{Derivative estimation problem}

Throughout this paper, $\mathbb{K}$ denotes a field of characteristic zero (usually $\mathbb{R}$ or $\mathbb{C}$ in many applications). Let $\mathbf{x}=\left(x_{1}, \ldots, x_{m}\right)$ be an element in $\mathbb{K}^{m}(m \in \mathbb{N})$. An $m$-tuple $N \in \mathbb{N}^{m}$ will be written as $N=\left(N_{1}, \ldots, N_{m}\right)$. We consider the partial order $\preceq$ on $\mathbb{N}^{m}$ defined by $N \preceq M$ if $N_{i} \leq M_{i}$, for all $1 \leq i \leq m$.

Let $f: U \subset \mathbb{R}^{m} \rightarrow \mathbb{R}$ be a multivariate signal where $U$ is some neighborhood of 0 . For a given $I=\left(i_{1}, \ldots, i_{m}\right) \in \mathbb{N}^{m}$, we denote $|I|:=i_{1}+\cdots+i_{m}, I !:=i_{1} ! \ldots i_{m} !$, $\mathbf{x}^{I}=x_{1}^{i_{1}} \ldots x_{m}^{i_{m}}$ and $\frac{\partial^{I}}{\partial \mathbf{x}^{I}}=\frac{\partial^{i_{1}}}{\partial x_{1}^{i_{1}}} \ldots \frac{\partial^{i_{m}}}{\partial x_{m}^{i_{m}}}$.

In practical problems, the available signal $f$ is usually corrupted by a noise. Denote by $f_{\bar{\sigma}}$ the noisy multivariate signal

$$
f_{\bar{\varpi}}(\mathbf{x})=f(\mathbf{x})+\varpi(\mathbf{x}),
$$

where $\varpi(\mathbf{x})$ is an additive noise. Assume that $f$ admits a Taylor series expansion at 0 and write:

$$
f(\mathbf{x})=\sum_{I \in \mathbb{N}^{m}} \frac{a_{I}}{I !} \mathbf{x}^{I}, \text { where } a_{I}=\frac{\partial^{I} f}{\partial \mathbf{x}^{I}}(0) .
$$


For $N=\left(N_{1}, \ldots, N_{m}\right) \in \mathbb{N}^{m}$, the truncated Taylor series $f_{N}$ at order $N$ is given by:

$$
f_{N}(\mathbf{x})=\sum_{I \preceq N} \frac{a_{I}}{I !} \mathbf{x}^{I} .
$$

The multivariate Laplace transform of a function $g: \mathbb{R}^{m} \rightarrow \mathbb{R}$ is given by:

$$
G(\mathbf{s}):=\mathcal{L}(g)(\mathbf{s})=\int_{\mathbb{R}_{+}^{m}} g(\mathbf{t}) \mathrm{e}^{-\mathbf{s}^{T} \mathbf{t}} d \mathbf{t},
$$

where $\mathbf{s}=\left(s_{1}, \ldots, s_{m}\right)$ is the Laplace (multi)variable and ${ }^{T} \mathbf{t}$ denotes the transpose of $\mathbf{t} \in \mathbb{R}^{m}$. That implies, for instance:

$$
\mathcal{L}\left(\frac{\mathbf{x}^{I}}{I !}\right)=\frac{1}{\mathbf{s}^{I+1}}
$$

where $\mathbf{s}^{I}=s_{1}^{i_{1}} \ldots s_{m}^{i_{m}}$. To realize $f_{N}(\mathbf{x})$ in the operational domain, we apply the Laplace transform (2) on (1). It results:

$$
F_{N}(\mathbf{s})=\sum_{I \preceq N} \frac{a_{I}}{\mathbf{s}^{I+1}} .
$$

For $\mathbf{x}, \mathbf{t} \in \mathbb{K}^{m}$, we use the notation:

$$
\int_{0}^{\mathbf{x}} g(\mathbf{t}) d \mathbf{t}=\int_{0}^{x_{1}} \ldots \int_{0}^{x_{m}} g\left(t_{1}, \ldots, t_{m}\right) d t_{1} \ldots d t_{m} .
$$

Recall that for a multivariate function $g$ and its Laplace transform $G$, the inverse Laplace transform satisfies

$$
\mathcal{L}^{-1}\left(\frac{1}{\mathbf{s}^{I}} \frac{\partial^{J} G}{\partial \mathbf{s}^{J}}\right)=\frac{1}{(I-\mathbf{1}) !} \int_{\mathbf{0}}^{\mathbf{x}}(\mathbf{x}-\tau)^{I-\mathbf{1}}(-\tau)^{J} g(\tau) d \tau
$$

where $\tau=\left(\tau_{1}, \ldots, \tau_{m}\right)$ and $\mathbf{1}=(1, \ldots, 1) \in \mathbb{K}^{m}$. For the sake of simplicity, we set:

$$
v_{I, J}=v_{I, J}(\tau)=(\mathbf{x}-\tau)^{I}(-\tau)^{J},
$$

and a shorter notation can be used:

$$
\mathcal{L}^{-1}\left(\frac{1}{\mathbf{s}^{I}} \frac{\partial^{J} G}{\partial \mathbf{s}^{J}}\right)=\frac{1}{(I-\mathbf{1}) !} \int_{\mathbf{0}}^{\mathbf{x}} v_{I-1, J}(\tau) g(\tau) d \tau .
$$

As we have seen in the introduction, a remarkable work on numerical differentiation by algebraic methods was written by Mboup et al. [20]. To illustrate their approach, we consider an example in the one-dimensional case. Consider the approximating polynomial function of degree $N$ of a real-valued signal $f(t)$, analytic on some time interval: 


$$
f(t)=\sum_{i=0}^{N} \frac{a_{i}}{i !} t^{i}
$$

originated from its Taylor series expansion, hence

$$
a_{i}=f^{(i)}(0), \forall 0 \leq i \leq N .
$$

The goal is to estimate the derivatives of the signal $f(t)$, that means, the coefficients $a_{i}$ in (7). Often, $f(t)$ will be assumed to be the measured signal from a signal $x(t)$ with some negligible noise, so we may consider only $f(t)$. For example, the estimation of the first derivative of $f(t)$ can be obtained in the following way: from the degree one polynomial

$$
f(t)=a_{0}+a_{1} t
$$

we obtain the operational domain expression given by the action of the Laplace transform. That yields:

$$
Y(s)=\frac{a_{0}}{s}+\frac{a_{1}}{s^{2}} .
$$

In [20], a minimal annihilator $\Pi$ is proposed to eliminate the term $a_{0}$. It consists of a suitable differential operator, in this case:

$$
\Pi=\frac{1}{s^{2}} \frac{d}{d s} s .
$$

(meaning that the expression is multiplied by $s$, then taking the derivative with respect to $s$ and finally multiplying by $\frac{1}{s^{2}}$ ). The term $a_{0}$ is eliminated after the action of $\Pi$. The time domain representation obtained thanks to the inverse Laplace transform provides an estimate $\widehat{\dot{f}}(t)$ of the first derivative $a_{1}=\dot{f}(0)$ :

$$
\widehat{\dot{f}}(t)=\frac{6}{T^{3}} \int_{0}^{T}(T-2 \tau) f(t-\tau) d \tau .
$$

(in practice, $f$ is replaced by its measure). The idea presented here is to individually estimate each derivative $a_{J}$ for $J \preceq N$. To formalize our procedure, we consider the following sets:

$$
\Theta=\left\{a_{I} \mid I \preceq N\right\}, \Theta_{\text {est }}=\left\{a_{J}\right\} \text { and } \Theta_{\text {est }}=\Theta \backslash \Theta_{\text {est }} .
$$

The definition of $\Theta, \Theta_{\text {est }}$ and $\Theta_{\text {est }}$ is clear: $\Theta$ contains all the parameters, $\Theta_{\text {est }}$ contains the parameters to be estimated and $\Theta_{\text {est }}$ the remaining ones. The relation $(\mathcal{R})$ below follows from (3):

$$
\mathcal{R}: P(\mathbf{s}) F_{N}(\mathbf{s})+Q(\mathbf{s})+\bar{Q}(\mathbf{s})=0
$$

where 


$$
\begin{aligned}
& P(\mathbf{s})=\mathbf{s}^{N}, Q(\mathbf{s})=-a_{J} \mathbf{s}^{N-J-1} \in \mathbb{K}_{\Theta_{\mathrm{est}}}\left[\mathbf{s}, \frac{1}{\mathbf{s}}\right] \text { and } \\
& \bar{Q}(\mathbf{s})=-\sum_{I \preceq N, I \neq J} a_{I} \mathbf{s}^{N-I-1} \in \mathbb{K}_{\Theta_{\overline{\mathrm{est}}}}\left[\mathbf{s}, \frac{1}{\mathbf{s}}\right]
\end{aligned}
$$

By $\mathbb{K}_{\Theta_{\text {est }}}$ and $\mathbb{K}_{\Theta_{\text {est }}}$, we denote respectively the algebraic extensions $\mathbb{K}_{\Theta_{\text {est }}}=$ $\mathbb{K}\left(\Theta_{\text {est }}\right)$ and $\mathbb{K}_{\Theta_{\overline{e s t}}}=\mathbb{K}\left(\Theta_{\text {est }}\right)$.

Based on the relation $\mathcal{R}(8)$, three polynomials $P, Q$ and $\bar{Q}$ are defined taking into account the coefficients to be identified: $P$ is the polynomial multiplying $F_{N}(\mathbf{s})$, $Q$ contains the coefficient to be estimated, while $\bar{Q}$ is formed by all the remaining terms. To obtain an equation containing only known terms and $a_{J}$, the polynomial $\bar{Q}$ must be eliminated. That will provide a formula for the estimate of $a_{J}$.

To annihilate $\bar{Q}$, some particular differential operators must be chosen to act on $(\mathcal{R})$. These operators are called annihilators. Such algebraic estimators for $a_{J}$ will be constructed by using structural properties of the Weyl algebra (see the Appendix).

Let us stress that if $\Pi$ is an annihilator estimating $a_{J}$, the partial derivative of $f$ at any other point $\mathbf{p} \in \mathbb{K}^{m}$ can be obtained by computing $\Pi(\mathcal{L}(f(\mathbf{x}+\mathbf{p})))$.

\subsection{Parameter estimation}

An example of parametric identification was given in the seminal paper by M. Fliess and H. Sira-Ramírez [10] and it concerns a first order input-output system:

$$
\dot{y}(t)=a y(t)+u(t)+\gamma_{0}
$$

where $a$ is a parameter to be identified and $\gamma_{0}$ is a constant perturbation. In the operational domain, thanks to the Laplace transform, the above equation becomes:

$$
s Y(s)-y(0)=a Y(s)+U(s)+\frac{\gamma_{0}}{s}
$$

where $s$ is the Laplace variable, $Y(s)$ and $U(s)$ denote the Laplace transform of $y(t)$ and $u(t)$ respectively. The action of the differential operator $\frac{1}{s^{2}} \frac{d^{2}}{d s^{2}} s$ on this expression yields:

$$
\begin{aligned}
\left(\frac{1}{s} \frac{d}{d s} Y(s)+\frac{2}{s^{2}} \frac{d^{2}}{d s^{2}} Y(s)\right) a=\frac{d^{2}}{d s^{2}} Y(s)+\frac{4}{s} \frac{d}{d s} Y(s)+\frac{2}{s^{2}} Y(s)- & \\
& \left(\frac{1}{s} \frac{d^{2}}{d s^{2}} U(s)+\frac{2}{s^{2}} \frac{d}{d s} U(s)\right) .
\end{aligned}
$$

Operational calculus rules yield the following estimation for $a$ : 


$$
a=\frac{\int_{0}^{1}\left(\left(2 v^{2}-3 v+1\right) v t u(t v)+\left(-6 v^{2}+6 v-1\right) y(t v)\right) d v}{t \int_{0}^{1} v\left(2 v^{2}-3 v+1\right) y(t v) d v}
$$

The parameter identification for a partial differential equation can be thought in a similar way. To illustrate this, a simple example of the one-dimensional heat equation is studied in the sequel. A similar algebraic approach was studied, for instance, in [38] for this same problem and in [11] for the parameter identification of a linear model of the planar motion of a heavy rope. The unidimensional Laplace transform was used in both these examples providing operational functions a as the solutions of an initial value problem. In this work, the Laplace transform in two variables is used to convert the partial differential equation into the operational domain representation.

\section{Annihilators via the Weyl algebra}

In the previous section, we have indicated that our aim is to annihilate the polynomial $\bar{Q}$ in the relation $\mathcal{R}(8)$, containing undesired parameters, see (9). That will be done by the action of annihilators: these are differential operators (or polynomials in the variables $\frac{\partial^{I}}{\partial s}$ ) with polynomial coefficients (or rational functions) in the Laplace variables $s_{1}, \ldots, s_{m}$. A practical realization of differential operators acting on polynomial variables is the Weyl algebra. So, this algebra appears naturally in this context and its structural properties will be quite useful in the choice of the annihilators.

This algebraic viewpoint is inspired by the work of M. Fliess et al. [9 10 19]. Details about the algebraic notions defined in the sequel can be found in the appendix and in [23,31] as well.

Next, we keep the notation defined in the appendix (see Section 7) to define the differential operators annihilators. They will help to construct algebraic estimators, either of derivatives or partial derivatives, or also of parameters.

Recall that $\mathrm{A}_{m}$ denotes the Weyl algebra $\mathrm{A}_{m}=\mathbb{K}[\mathbf{s}]\left[\frac{\partial}{\partial \mathbf{s}}\right]$ and $\mathrm{B}_{m}=\mathbb{K}(\mathbf{s})\left[\frac{\partial}{\partial \mathbf{s}}\right]$, respectively the polynomial rings in $\frac{\partial}{\partial \mathbf{s}}$ with coefficients in the polynomial ring $\mathbb{K}[\mathbf{s}]$ and in the fraction field $\mathbb{K}(\mathbf{s})$.

Definition 1. Let $R \in \mathbb{K}_{\Theta_{\text {est }}}\left[\mathbf{s}, \frac{1}{\mathrm{~s}}\right]$. A $R$-annihilator with respect to $\mathrm{B}_{m}$ is an element of $\mathrm{Ann}_{\mathrm{B}_{m}}(R)=\left\{F \in \mathrm{B}_{m} \mid F(R)=0\right\}$.

Consider $m \geq 2$. Let us remark that $\operatorname{Ann}_{\mathrm{B}_{m}}(R)$ is a left ideal of $\mathrm{B}_{m}$. Therefore, by Stafford's theorem (Theorem 2, Appendix), $\operatorname{Ann}_{\mathrm{B}_{m}}(R)$ is generated by two generators $\Pi_{1}$ and $\Pi_{2} \in \mathrm{B}_{m}$ :

$$
\operatorname{Ann}_{\mathrm{B}_{m}}(R)=\mathrm{B}_{m} \Pi_{1}+\mathrm{B}_{m} \Pi_{2} .
$$

We call the annihilators $\Pi_{1}$ and $\Pi_{2}$ minimal R-annihilators with respect to $\mathrm{B}_{m}$. The attribute minimal comes from the order of the differential operators. Notice that 
$\mathrm{Ann}_{\mathrm{B}_{m}}(R)$ contains annihilators in finite integral form, i.e. operators with coefficients in $\mathbb{K}\left[\frac{1}{\mathrm{~s}}\right]$.

Let us stress that thanks to the above Stafford's theorem, only two generators for the ideal are needed for a given $m \geq 2$.

Lemma 1. Consider $R(\mathbf{s})=\alpha \mathbf{s}^{N}=\alpha s_{1}^{n_{1}} \ldots s_{m}^{n_{m}}, N=\left(n_{1}, \ldots, n_{m}\right) \in \mathbb{Z}^{m}$ with $\alpha \in$ $\mathbb{K}_{\Theta_{\overline{e s t}}}$. A minimal R-annihilator is given by

$$
s_{i} \frac{\partial}{\partial s_{i}}-n_{i}, \forall 1 \leq i \leq m
$$

Recall that the degree of a monomial $\mathbf{s}^{I} \in \mathbb{K}\left[\mathbf{s}, \frac{1}{\mathbf{s}}\right]$ is $|I|$. The total degree of a polynomial in $\mathbf{s}$ is the maximum degree of its monomials.

Remark 1. Consider $R \in \mathbb{K}_{\Theta_{\text {est }}}\left[\mathbf{s}, \frac{1}{\mathbf{s}}\right]$ with a monomial $s^{I}$ of maximal degree. So $R$ has total degree $|I|$. Let $i_{k}=\max \left\{i_{j} \mid j=1, \ldots, m\right\}$. If $|I|>0$, then $\frac{\partial^{i_{k}+1}}{\partial s_{k}^{i_{k}+1}}$ is clearly an $R$-annihilator.

Now, recall that the polynomial to be annihilated in this differentiation problem is $(\operatorname{see}(9)$ ):

$$
\bar{Q}(\mathbf{s})=-\sum_{I \preceq N, I \neq J} a_{I} \mathbf{s}^{N-I-1} \in \mathbb{K}_{\Theta_{\overline{\mathrm{est}}}}\left[\mathbf{s}, \frac{1}{\mathbf{s}}\right] .
$$

By the previous remark, it results immediately:

Lemma 2. The differential operators $\frac{\partial^{n_{k}}}{\partial s_{k}{ }_{k}}$ are $\bar{Q}$-annihilators, for all $1 \leq k \leq m$.

To construct an alternative annihilator, an algorithm is sketched below:

\section{Algorithm 1}

Input: A polynomial $R=\sum_{\text {finite }} \mathbb{Z}_{I} b_{I} \mathbf{s}^{I}$ in $\mathbb{K}_{\Theta_{\overline{e s t}}}\left[\mathbf{s}, \frac{1}{\mathbf{s}}\right]$ of total degree $d \in \mathbb{N}$

Output: An R-annihilator

1. Set $\Pi=1 \in D$.

2. Choose a monomial of degree d in $R$, say $b_{J} \mathbf{S}^{J}$ with $J=\left(j_{1}, \ldots, j_{m}\right)($ so $|J|=d)$.

3. Choose $j_{k}=\min \left\{j_{\ell}>0 \mid \ell=1, \ldots, m\right\}$.

4. Apply $\pi=s_{k} \frac{\partial}{\partial s_{k}}-j_{k}$ (see Lemma 1 ) on $R$.

5. (a) If $\pi(R)=0$, then return $\pi$ and stop the algorithm.

(b) If $\pi(R) \neq 0$, then set $\Pi=\pi \circ \Pi$ and return to step (2) with $R \leftarrow \pi(R)$.

Example 1. Consider $m=2$ and the polynomial $R\left(s_{1}, s_{2}\right)=a_{00} s_{1}^{2} s_{2}+a_{01} s_{1}^{2}+a_{10} s_{1} s_{2}+$ $a_{20} s_{2} \in \mathbb{K}_{\Theta_{\overline{e s t}}}\left[s_{1}, s_{2}\right]$. A $R$-annihilator constructed with the above algorithm is $\left(s_{1} \frac{\partial}{\partial s_{1}}-2\right) \circ\left(s_{2} \frac{\partial}{\partial s_{2}}-1\right)$. 
The concept of an estimator must be defined in order to take into account the remaining terms in the relation $(\mathcal{R})$, after the action of an annihilator. Notice that the parameters to be estimated might appear in the set of coefficients of both polynomials $Q$ and $P$, but they might as well be present exclusively in one of the two. Therefore a $\bar{Q}$-annihilator must not eliminate all terms in $\Theta_{\text {est }}$, as formalized in the definition below:

Definition 2. An estimator $\pi \in \mathrm{B}$ is a $\bar{Q}$-annihilator satisfying

$$
\operatorname{coeffs}(\pi(\mathcal{R})) \cap \mathbb{K}_{\Theta_{\mathrm{est}}} \neq \emptyset,
$$

where coeffs $(R)$ denotes the set of coefficients of a polynomial $R \in \mathbb{K}_{\Theta}\left[\mathbf{s}, \frac{1}{\mathbf{s}}\right]$.

It is implied by this definition that the criterion on the coefficients must be considered in the choice of annihilators in the Algorithm 1.

It is important to stress that in some cases, it may be interesting to adopt another way of proceeding. For instance, several different annihilators can be constructed for each $\bar{Q}$. Then, Stafford's theorem can be applied to provide two minimal generators by using the package Stafford [30, 31]. The final step is to observe the criterion in Definition 2 in these generators to obtain an estimator.

\section{Derivative estimation}

To illustrate the algebraic method for numerical differentiation, we present here the estimation for a derivative in the two-dimensional case. Hence, the equation (1) is considered for $m=2$. For this example, we assume that the parameter to be estimated is $a_{21}=\frac{\partial^{3} f}{\partial x_{1}^{2} \partial x_{2}}(0,0)$. Based on (1), a truncated Taylor series at $N=(2,1)$ will then be used:

$$
f\left(x_{1}, x_{2}\right)=a_{00}+a_{10} x_{1}+a_{01} x_{2}+a_{20} x_{1}^{2}+a_{11} x_{1} x_{2}+a_{21} x_{1}^{2} x_{2} .
$$

The coefficient to be estimated is $a_{21}$ in the truncated Taylor series above, so we may distinguish the following polynomials $P, Q$ and $\bar{Q} \in \mathbb{K}\left[\mathbf{s}, \frac{1}{\mathbf{s}}\right]$ in the relation ( $\left.\mathcal{R}\right)$ $(\operatorname{see}(8)$ ):

$$
\begin{aligned}
& P\left(s_{1}, s_{2}\right)=s_{1}^{2} s_{2}, \\
& Q\left(s_{1}, s_{2}\right)=-a_{21} s_{1}^{-1} s_{2}^{-1} \in \mathbb{K}_{\Theta_{\text {est }}}\left[\mathbf{s}, \frac{1}{\mathbf{s}}\right] \text { and } \\
& \bar{Q}\left(s_{1}, s_{2}\right)=-\sum_{\substack{(i, j) \preceq N \\
(i, j) \neq(2,1)}} a_{i j} s_{1}^{1-i} s_{2}^{-j} \in \mathbb{K}_{\Theta_{\text {est }}}\left[\mathbf{s}, \frac{1}{\mathbf{s}}\right] .
\end{aligned}
$$


The first step of the estimation is to determine minimal $\bar{Q}$-annihilators. To begin, Lemma 1 helps to find two $\bar{Q}$-annihilators $\frac{\partial^{2}}{\partial s_{1}^{2}}\left(s_{1} \frac{\partial}{\partial s_{1}}+1\right)$ and $\frac{\partial}{\partial s_{2}}\left(s_{2} \frac{\partial}{\partial s_{2}}+1\right)$. However, they are not estimators since they clearly eliminate $Q$ as well and $Q$ is the only term in $\mathcal{R}$ with coefficients in $\mathbb{K}_{\Theta_{\overline{e s t}}}$ (see Definition 2 .

We then follow the Algorithm 1 to determine an alternative $\bar{Q}$-annihilator that may also be a estimator. In the case of $a_{21}$, we obtain:

$$
\Pi=\frac{1}{s_{1}^{2} s_{2}} \frac{\partial^{2}}{\partial s_{1} s_{2}}\left(s_{1} \frac{\partial}{\partial s_{1}}-1\right) .
$$

Let us remark that for other coefficients $a_{k \ell}$, some annihilators are proposed in [36] and in [44] as well. Using Remark44 in the Appendix, it can be shown that the annihilator $\Pi$ is a minimal annihilator.

The action of $\Pi$ on the relation $\mathcal{R}$ with $P, Q$ and $\bar{Q}$ defined above, provides the following expression:

$$
\begin{aligned}
2 \frac{F\left(s_{1}, s_{2}\right)}{s_{1}{ }^{3} s_{2}{ }^{2}}+4 \frac{\frac{\partial}{\partial s_{1}} F\left(s_{1}, s_{2}\right)}{s_{1}{ }^{2} s_{2}{ }^{2}}+2 \frac{\frac{\partial}{\partial s_{2}} F\left(s_{1}, s_{2}\right)}{s_{1}{ }^{3} s_{2}} & +4 \frac{\frac{\partial^{2}}{\partial s_{2} \partial s_{1}} F\left(s_{1}, s_{2}\right)}{s_{1}{ }^{2} s_{2}}+\frac{\frac{\partial^{2}}{\partial s_{1}{ }^{2}} F\left(s_{1}, s_{2}\right)}{s_{1} s_{2}{ }^{2}} \\
& +\frac{\frac{\partial^{3}}{\partial s_{2} \partial s_{1}{ }^{2}} F\left(s_{1}, s_{2}\right)}{s_{1} s_{2}}+2 \frac{a_{2,1}}{s_{1}{ }^{6} s_{2}{ }^{4}}=0 .
\end{aligned}
$$

Isolating the term with $a_{21}$ and applying the inverse Laplace transform (6) provides the consequent estimate:

$$
a_{21}=-\frac{360}{x_{1}^{5} x_{2}^{3}} \int_{\mathbf{0}}^{\left(x_{1}, x_{2}\right)}\left(v_{2,1,0,0}+v_{0,1,2,0}-4 v_{1,1,0,0}-4 v_{1,0,0,1}+v_{2,0,0,1}+v_{0,0,2,1}\right) f(\tau) d \tau,
$$

where we use the notation 5 :

$$
v_{I, J}=\left(x_{1}-\tau\right)^{i_{1}}\left(x_{2}-\eta\right)^{i_{2}}(-\tau)^{j_{1}}(-\eta)^{j_{2}},
$$

for all $I=\left(i_{1}, i_{2}\right), J=\left(j_{1}, j_{2}\right) \in \mathbb{N}^{2}$.

\section{Parameter estimation}

In the previous section, we examined the case of numerical differentiation where annihilators were used to eliminate the undesired terms of the truncated Taylor series seen in the operational domain. Moreover, as we have seen in the introduction, a similar procedure may provide estimates for parameters in an ordinary differential equation.

In this section, we present a parameter identification problem for a two-dimensional partial differential equation. The following classical example was studied in [38 44], for instance. Consider the problem of the heat conduction in a thin rod of length 1 . 
Let $w:(z, t) \mapsto w(z, t)$ be the function representing the temperature at position $z$ at time $t$. The partial differential equation describing this problem is given by:

$$
\frac{\partial^{2}}{\partial z^{2}} w(z, t)-\beta \frac{\partial}{\partial t} w(z, t)-\alpha w(z, t)=0
$$

The rod is assumed to be perfectly isolated at $z=0$, so $\frac{\partial}{\partial z} w(0, t)=0$. The condition at $z=1$ is not of interest to us and we assume that the initial temperature is 0 . In addition, we suppose that the temperature $w(z, t)$ at any time $t$ and position $z$ at the rod can be measured and used in the parameter estimation. To simplify the notation, we write $q_{0}: t \mapsto w(0, t)$ and $f: t \mapsto w(z, 0)$.

The goal is to identify the parameters $\alpha$ and $\beta$. The algebraic method used in the previous subsection is applied here. Using the notation $r: t \mapsto \frac{\partial}{\partial z} w(0, t)$, the Laplace transform (2) is employed to realize the partial differential equation (10) as an algebraic equation in the Laplace variable $\mathbf{s}=\left(s_{1}, s_{2}\right)\left(s_{1}\right.$ corresponds to $z$ and $s_{2}$ corresponds to $t$ ):

$$
\left(s_{1}^{2}-\beta s_{2}-\alpha\right) W\left(s_{1}, s_{2}\right)+\beta F\left(s_{1}\right)-s_{1} Q_{0}\left(s_{2}\right)-R\left(s_{2}\right)=0,
$$

where $W(\mathbf{s}), F\left(s_{1}\right), Q_{0}\left(s_{2}\right)$ and $R\left(s_{2}\right)$ denote the Laplace transforms of $w(z, t)$ with respect to $z$ and $t$, of $f(z)$ with respect to $z$, and of $q_{0}(t)$ and $r(t)$ with respect to $t$ respectively. Since by hypothesis, $R \equiv 0$ and $F \equiv 0$, the equation (11) leads to:

$$
\left(-\beta s_{2}+s_{1}^{2}-\alpha\right) W\left(s_{1}, s_{2}\right)-s_{1} Q_{0}\left(s_{2}\right)=0 .
$$

Here the set of parameters $\Theta_{\text {est }}$ to be estimated is

$$
\Theta_{\mathrm{est}}=\{\alpha, \beta\},
$$

while $\Theta_{\mathrm{est}}=\emptyset$. Following the procedure described at the beginning of Section 2, a system on the indeterminates $\alpha$ and $\beta$ will be determined by acting suitable annihilators on (12). So a two-steps procedure will be applied. In the first step, we rewrite (12) in the form of a $\mathcal{R}$-relation (see (8)):

$$
\mathcal{R}: P(\mathbf{s}) W(\mathbf{s})+Q(\mathbf{s})+\bar{Q}(\mathbf{s})=0,
$$

where

$$
P(\mathbf{s})=s_{1}^{2}-\beta s_{2}-\alpha, Q(\mathbf{s})=-s_{1} Q\left(s_{2}\right) \text { and } \bar{Q}(\mathbf{s})=0 .
$$

As mentioned before, the problem of annihilating $\bar{Q}$ is tackled by finding suitable $\bar{Q}$-annihilators that will lead to a system in $\Theta_{\text {est }}$. Notice in this example that the parameters to be estimated also appear in the coefficients of the polynomial $P$.

Here, the polynomial $\bar{Q}$ to be annihilated in 14 is 0 , so we may consider the above equation (14). In order to apply the inverse Laplace transform (4), we divide this equation by $s_{1}^{3} s_{2}^{2}$. Using the notation $v_{I, J}=(z-\tau)^{i_{1}}(t-\eta)^{i_{2}}(-\tau)^{j_{1}}(-\eta)^{j_{2}}$, for all $I=\left(i_{1}, i_{2}\right), J=\left(j_{1}, j_{2}\right) \in \mathbb{N}^{2}$, we obtain in the spatial domain: 


$$
A_{11} \alpha+A_{12} \beta=B_{1}
$$

where

$$
\left\{\begin{array}{l}
A_{11}=-\frac{1}{2} \int_{0}^{(z, t)} v_{2,1,0,0} w(\tau, \eta) \mathrm{d} \tau \mathrm{d} \eta \\
A_{12}=-\frac{1}{2} \int_{0}^{(z, t)} v_{2,0,0,0} w(\tau, \eta) \mathrm{d} \tau \mathrm{d} \eta \\
B_{1}=-\int_{0}^{(z, t)} v_{0,1,0,0}\left(w(\tau, \eta)-q_{0}(\eta)\right) \mathrm{d} \tau \mathrm{d} \eta
\end{array}\right.
$$

In the second step, we will try to eliminate the term with the polynomial $Q_{0}$ : in this case, the polynomial $\bar{Q}$ to be annihilated in $\sqrt{14}$ is $\bar{Q}(\mathbf{s})=-s_{1} Q_{0}\left(s_{2}\right)$ while $Q(\mathbf{s})=\beta F\left(s_{1}\right)$. We propose the $\bar{Q}$-annihilator $\pi=\frac{\partial^{2}}{\partial s_{1}^{2}}$. Applying $\pi$ on the relation (13) gives:

$$
-\alpha \frac{\partial^{2}}{\partial s_{1}^{2}} W+\left(\frac{\mathrm{d}^{2}}{\mathrm{~d} s_{1}^{2}} F\left(s_{1}\right)-s_{2} \frac{\partial^{2}}{\partial s_{1}^{2}} W\right) \beta+s_{1}^{2} \frac{\partial^{2}}{\partial s_{1}^{2}} W+4 s_{1} \frac{\partial}{\partial s_{1}} W+2 W=0 .
$$

After dividing the above equation by a suitable monomial in $\mathbf{s}$, namely $s_{1}^{2} s_{2}^{2}$, we obtain:

$$
\left\{\begin{array}{l}
A_{21}=-\frac{1}{2} \int_{0}^{(z, t)} v_{2,1,0,2} w(\tau, \eta) \mathrm{d} \tau \mathrm{d} \eta \\
A_{22}=-\frac{1}{2} \int_{0}^{(z, t)} v_{2,0,0,2} w(\tau, \eta) \mathrm{d} \tau \mathrm{d} \eta \\
B_{2}=\int_{0}^{(z, t)}\left(4 v_{1,1,0,0}-v_{2,1,0,0}-v_{0,1,2,0}\right) w(\tau, \eta) \mathrm{d} \tau \mathrm{d} \eta
\end{array}\right.
$$

A system on $\Theta_{\text {est }}$ results from the actions of $\bar{Q}$-annihilators:

$$
\left(\begin{array}{ll}
A_{11} & A_{12} \\
A_{21} & A_{22}
\end{array}\right)\left(\begin{array}{l}
\alpha \\
\beta
\end{array}\right)=\left(\begin{array}{l}
B_{1} \\
B_{2}
\end{array}\right)
$$

Solving the system provides the estimates of $\alpha$ and $\beta$.

Remark 2. In [44], other annihilators were proposed since the statement of the problem and its initial and boundary conditions were different. Again, recall that a very special property of the two-dimensional Weyl algebra is Stafford's theorem (see Theorem 28. This important result allows the computation of two minimal annihila- 
tors. Moreover the package Stafford [30,31] uses a highly efficient algorithm to calculate these differential operators.

\section{Conclusion}

In this paper, we provided a short preview on algebraic estimation for derivatives and for parameters in linear systems. Advantages and possible drawbacks of this algebraic framework were evoked in a brief state-of-art. More detailed problem statements were given in the subsequent sections, followed by proposed solutions within the algebraic context. The algebraic properties in the appendix, notably concerning the Weyl algebra, support these solutions. Furthermore, we illustrate our algebraic method with two typical examples: in the case of two-dimensional numerical differentiation, while in the case of parameter estimation for partial derivatives systems, the thin rod example is studied. An essential point deserves to be emphasized: the algebraically obtained estimated are based on integrals of measured signals. These particular integrals play the role of time-varying filters. Furthermore, closed formulas for derivatives and parameters estimates that obtained with our method, via the Weyl algebra tools, are presented in this paper.

\section{Appendix}

We recall below some basic definitions and properties of the Weyl algebra.

Definition 3. Let $m \in \mathbb{N} \backslash\{0\}$. The Weyl algebra $\mathrm{A}_{m}(\mathbb{K})$ (or $\mathrm{A}_{m}$ ) is the $\mathbb{K}$-algebra with generators $p_{1}, q_{1}, \ldots, p_{m}, q_{m}$ and relations

$$
\left[p_{i}, q_{j}\right]=\delta_{i j},\left[p_{i}, p_{j}\right]=\left[q_{i}, q_{j}\right]=0, \forall 1 \leq i, j \leq m
$$

where $[\cdot, \cdot]$ is the commutator defined by $[u, v]:=u v-v u$, for all $u, v \in \mathrm{A}_{m}(\mathbb{K})$.

The Weyl algebra $A_{m}$ can be realized as the algebra of polynomial differential operators on the polynomial ring $\mathbb{K}[\mathbf{s}]$ by setting:

$$
p_{i}=\frac{\partial}{\partial s_{i}} \text { and } q_{i}=s_{i} \times, \forall 1 \leq i \leq m,
$$

where $\times$ denotes the multiplication map. That implies that $A_{m}$ can be written as $\mathrm{A}_{m}=\mathbb{K}[\mathbf{q}][\mathbf{p}]=\mathbb{K}[\mathbf{s}]\left[\frac{\partial}{\partial \mathbf{s}}\right]$. The algebra of differential operators $\mathrm{B}_{m}(\mathbb{K})$ (or $\mathrm{B}_{m}$ ) on $\mathbb{K}[\mathbf{s}]$ with coefficients in the rational functions field $\mathbb{K}(\mathbf{s})$ is naturally related to $\mathrm{A}_{m}(\mathbb{K})$. In this case, we can write $\mathrm{B}_{m}:=\mathbb{K}(\mathbf{q})[\mathbf{p}]=\mathbb{K}(\mathbf{s})\left[\frac{\partial}{\partial \mathbf{s}}\right]$. 
A $\mathbb{K}$-basis for $\mathrm{A}_{m}$ is given by $\left\{\mathbf{q}^{I} \mathbf{p}^{J} \mid I, J \in \mathbb{N}^{m}\right\}$ where $\mathbf{q}=q_{1}^{i_{1}} \ldots q_{m}^{i_{m}}$ and $\mathbf{p}=$ $p_{1}^{i_{1}} \ldots p_{m}^{i_{m}}$. An operator $F \in \mathrm{A}_{m}$ can be written in a canonical form,

$$
F=\sum_{I, J} \lambda_{I J} \mathbf{q}^{I} \mathbf{p}^{J} \text { with } \lambda_{I J} \in \mathbb{K} .
$$

Similarly, an element $F \in \mathrm{B}_{m}$ can be written as

$$
F=\sum_{I} g_{I}(\mathbf{s}) \frac{\partial^{I}}{\partial s^{I}}, \text { where } g_{I}(\mathbf{s}) \in \mathbb{K}(\mathbf{s}) .
$$

The order of $F$ is defined as $\operatorname{ord}(F)=\max \left\{\left[I|| g_{I}(\mathbf{s}) \neq 0\right\}\right.$. This definition holds for the Weyl algebra $A_{m}$ as well, since $A_{m} \subset B_{m}$. Some useful properties of $A_{m}$ and $\mathrm{B}_{m}$ are given by the following propositions (see for instance [7]):

Proposition 1. The algebra $\mathrm{A}_{m}$ is a domain. Moreover, $\mathrm{A}_{m}$ is a simple algebra (i.e. it contains no nontrivial ideals) and also a left Noetherian ring (i.e. every left ideal is finitely generated).

These properties are shared by $B_{m}$. In addition, $A_{m}$ is neither a principal right domain, nor a principal left domain. Nevertheless this is true for $B_{1}$ :

Proposition 2. $\mathrm{B}_{1}$ admits a left division algorithm, that is, if $F, G \in \mathrm{B}_{1}$, then there exists $Q, R \in \mathrm{B}_{1}$ such that $F=Q G+R$ and $\operatorname{ord}(R)<\operatorname{ord}(G)$. Consequently, $\mathrm{B}_{1}$ is a principal left domain.

Alas, this proposition does not hold for $\mathrm{B}_{m}$ for $m \geq 2$. But an important theorem by T. Stafford (see [40]) provides an remarkable property on the number of generators of a left ideal in the Weyl algebra. Namely, Stafford proved that every left ideal of $D\left(D=\mathrm{A}_{m}\right.$ or $\left.\mathrm{B}_{m}\right)$ can be generated by two elements in $D$ :

Theorem 2 (Stafford). Let $\mathfrak{a}$ be a left ideal of $D$ generated by three elements $F_{1}, F_{2}$ and $F_{3} \in D$. Then, there exist $G_{1}$ and $G_{2} \in D$ such that

$$
\mathfrak{a}=D\left(F_{1}+G_{1} F_{3}\right)+D\left(F_{2}+G_{2} F_{3}\right) .
$$

An effective implementation in Maple, named Stafford, of this important theorem can be found in the work of A. Quadrat and D. Robertz [30].

Remark 3. It is important to notice that the principality of $\mathrm{B}_{1}$ was largely used in the initial works on algebraic methods applied to univariate numerical differentiation, such as [20] or parameter estimation in ordinary differential equations, see for instance [47]. In the multivariate case, the principality holds no longer, therefore the importance of Stafford's theorem.

To close this part, we remark a useful identity:

Remark 4. For arbitrary $N, M \in \mathbb{N}^{r}$, we have 


$$
\frac{\partial^{N}}{\partial \mathbf{s}^{N}} \frac{1}{\mathbf{s}^{M}}=\sum_{0 \preceq J \preceq N}\left(\begin{array}{l}
N \\
J
\end{array}\right)(-1)^{|N-J|} \frac{M^{\overline{N-J}}}{\mathbf{s}^{M+N-J}} \frac{\partial^{J}}{\partial \mathbf{s}^{J}},
$$

where $\left(\begin{array}{c}N \\ J\end{array}\right)=\left(\begin{array}{c}n_{1} \\ j_{1}\end{array}\right) \ldots\left(\begin{array}{c}n_{r} \\ j_{r}\end{array}\right), M^{\bar{N}}=m_{1}^{\overline{n_{1}}} \ldots m_{r}^{\overline{n_{r}}}$ and $m_{i}^{\overline{n_{i}}}$ denotes the rising factorial $\left(m_{i}^{\overline{n_{i}}}=m_{i}\left(m_{i}+1\right) \ldots\left(m_{i}+n_{i}-1\right)\right)$.

\section{References}

1. M. Al-Alaoui, "A class of second-order integrators and low-pass differentiators," IEEE Transactions on Circuits and Systems I: Fundamental Theory and Applications, vol. 42, no. 4, pp. 220-223, Apr 1995. 2

2. B. Carlsson, A. Ahln, and M. Sternad, "Optimal differentiation based on stochastic signal models," IEEE Transactions on Signal Processing, vol. 39, pp. 341-353, 1991. 1

3. C.-K. Chen and J.-H. Lee, "Design of high-order digital differentiators using $L_{1}$ error criteria," IEEE Transactions on Circuits and Systems II: Analog and Digital Signal Processing, vol. 42, no. 4, pp. 287-291, Apr 1995. 2

4. Y. Chitour, "Time-varying high-gain observers for numerical differentiation," IEEE Transactions on Automatic Control, vol. 47, no. 9, pp. 1565-1569, Sep 2002. 1

5. L. Coluccio, A. Eisinberg, and G. Fedele, "A property of the elementary symmetric functions on the frequencies of sinusoidal signals," Signal Processing, vol. 89, no. 5, pp. $765-777$, 2009. 3

6. A. M. Dabroom and H. K. Khalil, "Discrete-time implementation of high-gain observers for numerical differentiation," International Journal of Control, vol. 72, no. 17, pp. 1523-1537, 1999. 1

7. J. Dixmier, Algèbres enveloppantes. Gauthier-Villars, 1974. 15

8. D. Efimov and L. Fridman, "A hybrid robust non-homogeneous finite-time differentiator," IEEE Transactions on Automatic Control, vol. 56, no. 5, pp. 1213-1219, 2011.2

9. M. Fliess, M. Mboup, H. Mounier, and H. Sira-Ramírez, "Questioning some paradigms of signal processing via concrete examples," in in Algebraic Methods in Flatness, Signal Processing and State Estimation, G. S.-N. H. Sira-Ramírez, Ed. Editiorial Lagares, 2003, pp. 1-21. 28

10. M. Fliess and H. Sira-Ramírez, "An algebraic framework for linear identification," ESAIM Control Optim. Calc. Variat., vol. 9, pp. 151-168, 2003. 2, 3, 7 , 8

11. N. Gehring, T. Knppel, J. Rudolph, and F. Woittennek, "Algebraic identification of heavy rope parameters," in Proc. 16th IFAC Symposium on System Identification, July 2012, pp. 161-166. 8

12. M. Hou, "Parameter identification of sinusoids," IEEE Transactions on Automatic Control, vol. 57, no. 2, pp. 467-472, February 2012. 3

13. S. Ibrir, "On observer design for nonlinear systems," Intern. J. Syst. Sci., vol. 37, no. 15, pp. 1097-1109, Dec. 2006. 1

14. __ "Linear time-derivative trackers," Automatica, vol. 40, no. 3, pp. 397 - 405, 2004. 1

15. S. Ibrir and S. Diop, "A numerical procedure for filtering and efficient high-order signal differentiation," International Journal of Applied Mathematics and Computer Science, vol. 14, no. 2, p. 201208, 2004. 1

16. A. Levant, "Robust exact differentiation via sliding mode technique*," Automatica, vol. 34, no. 3, pp. $379-384,1998$. 2

17. — "Higher-order sliding modes, differentiation and output-feedback control," International Journal of Control, vol. 76, no. 9-10, pp. 924-941, 2003. 1

18. D. Liu, "Analyse d'erreurs d'estimateurs des dérivées de signaux bruités et applications," Ph.D. dissertation, 2011. 2

19. M. Mboup, "Parameter estimation for signals described by differential equations," Applicable Analysis, vol. 88, pp. 29-52, 2009. 28 
20. M. Mboup, C. Join, and M. Fliess, "Numerical differentiation with annihilators in noisy environement," Numerical Algorithms, vol. 50, pp. 439-467, 2009. 2 23, 5 6 6

21. M. Mboup, "Parameter estimation via differential algebra and operational culculus," Research Report, 2007. 3

22. _ - "Neural spike detection and localisation via Volterra filtering," in 22nd IEEE Workshop on Machine Learning for Signal Processing, Santander, Spain, Sep. 2012. 2

23. J. McConnell and J. Robson, Noncommutative Noetherian Rings, A. M. Soc., Ed. Hermann, 2000. 8

24. L. Menhour, B. d'Andrea Novel, C. Boussard, M. Fliess, and H. Mounier, "Algebraic nonlinear estimation and flatness-based lateral/longitudinal control for automotive vehicles," in Intelligent Transportation Systems (ITSC), 2011 14th International IEEE Conference on, Oct 2011, pp. 463-468. 2

25. J. Mikusiński and T. Boehme, Operational calculus, ser. International series of monographs in pure and applied mathematics. Pergamon Press, 1987. 4

26. J. Mikusiński, Operational calculus. Pergamon Oxford, 1959. 4

27. R. Morales, J. Somolinos, and H. Sira-Ramírez, "Control of a DC motor using algebraic derivative estimation with real time experiments," Measurement, vol. 47, pp. 401 - 417, 2014. 2

28. R. Morales, F. Rincn, J. D. Gazzano, and J. C. Lopez, "Real-time algebraic derivative estimations using a novel low-cost architecture based on reconfigurable logic," Sensors, vol. 14, no. 5 , p. $9349,2014.2$

29. W. Perruquetti, T. Floquet, and E. Moulay, "Finite-time observers: application to secure communication," Automatic Control, IEEE Transactions on, vol. 53, no. 1, pp. 356-360, 2008. 2

30. A. Quadrat and D. Robertz, "Computation of bases of free modules over the weyl algebras," J. Symbolic Comput., vol. 42, pp. 1113-1141, 2007. 1014

31. A. Quadrat, "An introduction to constructive algebraic analysis and its applications," INRIA, Research Report RR-7354, Jul. 2010. [Online]. Available: https://hal.inria.fr/inria-00506104 810,14

32. C. Rader and L. Jackson, "Approximating noncausal iir digital filters having arbitrary poles, including new hilbert transformer designs, via forward/backward block recursion," IEEE Transactions on Circuits and Systems I: Regular Papers, vol. 53, no. 12, pp. 2779-2787, Dec 2006. 2

33. J. Reger, P. Mai, and H. Sira-Ramirez, "Robust algebraic state estimation of chaotic systems," in Computer Aided Control System Design, 2006 IEEE International Conference on Control Applications, 2006 IEEE International Symposium on Intelligent Control, 2006 IEEE, Oct 2006, pp. 326-331.2

34. S. Riachy, Y. Bachalany, M. Mboup, and J.-P. Richard, "An algebraic method for multidimensional derivative estimation," in MED'08, 16th IEEE Mediterranean Conference on Control and Automation. Ajaccio, Corsica, France: IEEE, 2008. 3

35. — - "Différenciation numérique multivariable I : estimateurs algébriques et structure," in Sixième Conférence Internationale Francophone d'Automatique Nancy, France, 2-4 juin 2010, Nancy, France, 2010. 3

36. S. Riachy, M. Mboup, and J.-P. Richard, "Multivariate numerical differentiation," JCAM, Aug. 2011. 311

37. R. A. Roberts and C. T. Mullis, Digital Signal Processing. Reading, MA: Addison-Wesley, 1987. 2

38. J. Rudolph and F. Woittennek, "An algebraic approach to parameter identification in linear infinite dimensional systems," in Proc. 16th Mediterranean Conference on Control and Automation, june 2008, pp. 332-337. 3 . 11

39. H. Sira-Ramírez, C. G. Rodríguez, J. C. Romero, and A. L. Juárez, Algebraic Identification and Estimation Methods in Feedback Control Systems, 1st ed. Wiley Publishing, 2014. 2

40. J. T. Stafford, "Module structure of Weyl algebras," J. London Math. Soc., vol. 18, no. 3, pp. 429-442, 1978. 15 
41. Y. Su, C. Zheng, P. Mueller, and B. Duan, "A simple improved velocity estimation for lowspeed regions based on position measurements only," Control Systems Technology, IEEE Transactions on, vol. 14, no. 5, pp. 937-942, Sept 2006. 1

42. J. Trapero, H. Sira-Ramírez, and V. Battle, "A fast on-line frequency estimator of lightly damped vibrations in flexible structures," J. Sound Vibration, vol. 307, pp. 365-378, 2007. 2

43. J. Trapero-Arenas, M. Mboup, E. Pereira-Gonzalez, and V. Feliu, "On-line frequency and damping estimation in a single-link flexible manipulator based on algebraic identification," in Control and Automation, 2008 16th Mediterranean Conference on, june 2008, pp. 338-343. 2

44. R. Ushirobira, A. Korporal, and W. Perruquetti, "On an algebraic method for derivatives estimation and parameter estimation for partial derivatives systems," in Mathematical Theory of Networks and Systems, Groningen, Netherlands, Jul. 2014. 11.13

45. R. Ushirobira, W. Perruquetti, M. Mboup, and M. Fliess, "Estimation algébrique des paramètres intrinsèques d'un signal sinusoïdal biaisé en environnement bruité," in Proc. Gretsi, Bordeaux, France, Sep. 2011. 3

46. — - "Algebraic parameter estimation of a biased sinusoidal waveform signal from noisy data," in Sysid 2012, 16th IFAC Symposium on System Identification, Brussels, Belgique, 2012. 3

47. $\quad$, "Algebraic parameter estimation of a multi-sinusoidal waveform signal from noisy data," in European Control Conference, Zurich, Suisse, Apr. 2013. 3.15

48. X. Xia, "Global frequency estimation using adaptive identifiers," vol. 47, no. 7, pp. 11881193, jul 2002. 3

49. K. Yosida, Operational calculus: a theory of hyperfunctions. Springer-Verlag New York, 1984. 4

50. L. Yu, J.-P. Barbot, and T. Floquet, "Tire/Road contact condition identification using algebraic numerical differentiation," in IFAC Symposium on System Identification, SYSID, Saint-Malo, France, 2009. 2 